\title{
Access Control Scheme based on Preemption for Integrated Video/Data CDMA Systems
}

\author{
Jae-Woo So†, Young-uk Chungł, and Dong-Ho Cho $\ddagger$ \\ † IP One, Inc., 1004, Daechi-dong, Gangnam-gu, Seoul 135-851, Republic of Korea \\ E-mail: israin@ipone.co.kr, TEL: +82-2-2222-4634, FAX: +82-2222-4639 \\ $\ddagger$ Dept. of EECS, KAIST, Daejeon 305-701, Republic of Korea \\ E-mail: thatfox@comis.kaist.ac.kr, dhcho@ee.kaist.ac.kr \\ TEL: +82-42-869-8067, FAX: +82-42-867-0550
}

\begin{abstract}
The third-generation mobile communication system is being developed to support to a wide range of services with different bit rates such as data, video as well as voice. We propose an access control scheme based on preemption policy to provide efficiently high priority video services and low priority data services. We analyze the proposed scheme using Markov model and present the performance evaluation of video service and data service in view of video packet dropping probability and data message delay.
\end{abstract}

\section{INTRODUCTION}

Traditional second generation cellular code division multiple access (CDMA) networks mainly support voice services and low-bit-rate data serves, in which the data is transmitted on the dedicated channel with a fixed rate. However, many researches have shown that it is insufficient to apply the current cellular CDMA architecture (e.g., fixed rate, high processing gain) directly to multimedia services. The bursty nature of multimedia transmissions, such as video and web surfing, requires stronger statistical multiplexing and flexible resource management. In order to offer multimedia services with different bit-rate and quality-of-service, the schemes sharing radio network resources among many users in a flexible and efficient way have been studied in third generation communication systems [1]-[8].

The integration of voice and data service in the same wireless packet network has been studied in [1]-[4]. In [1] and [2], voice communications and data communications are provided over common radio channel. They both use preemption policy for data transmissions and therefore present that the packet dropping probability for voice communications is independent of data transmissions. In [3], the output and delay process of integrated voice/data slotted CDMA network systems with random access protocol for packet radio communications are analyzed. In this system, the allocation of codes to voice communication has higher priorities over that to data communications. Using a discrete-time Markov process, they derived the throughput, the average delay of data packet, and the blocking probability of voice calls. In [4], the benefits of using access control for data in an integrated voice/data CDMA system are evaluated. They achieved an improved capacity for data at the expense of introducing delay for data packets by scheduling the transmission of data packets in periods of low voice activity. In [5]-[8], the multiple access techniques for accommodating the multimedia services in wireless CDMA communication networks are studied. In [5], a medium access control (MAC) protocol for a cellular packet CDMA carrying multirate traffic is proposed. They adopted a packet-oriented MAC protocol with prioritized queuing and assumed that the unit of transmitting packet is ATM cell. An analytical model was developed for solving the equilibrium state associated with the protocol. Numerical evaluation in terms of throughput efficiency and delay has been presented for two-type traffic, high rate traffic and low rate traffic. In [6], the problem of call admission control (CAC) in a multimedia CDMA system is investigated. They asserted that CAC functions should be included in the system since even a small number of high-bit-rate users can degrade the low-bit-rate users' performance and considerably increase their outage probability. In [7], a medium access protocol is proposed to provide constantbit-rate (voice), variable bit-rate (video), and low priority data services. Their protocols are based on the reservation multiple access scheme and integrate voice, video, and data traffic in the same packet radio network, using a constant or variable bit-rate transmission.

In this paper, we propose an access control scheme based on preemption policy in integrated video/data CDMA systems. Both video communications and data communications are provided over the common radio channel to use efficiently radio resource. We analyze the proposed scheme and compare the performance of the proposed scheme with that of simple channel assignment (SCA) scheme without preemption policy in view of video packet loss probability and data message delay.

The remainder of this paper is organized as follows. In Section II, we describe the system model and traffic models. In Section III, we explain the SCA scheme without preemption policy and analyze the system performance by using Markov process in view of video packet dropping probability and data message delay. In Section IV, we propose a channel assignment scheme with preemption (CAP) for data transmissions and describe the proposed scheme in detail. Also, the performance of the proposed scheme is evaluated. In Section V, numerical results are provided and concluding remarks are presented in Section VI.

\section{System ModeL}

Both $N_{v d}$ video terminals (VDT's) and $N_{d}$ data terminals (DT's) are assumed to access the uplink channel of a microcellar network. Video traffic and data traffic are considered as real- 


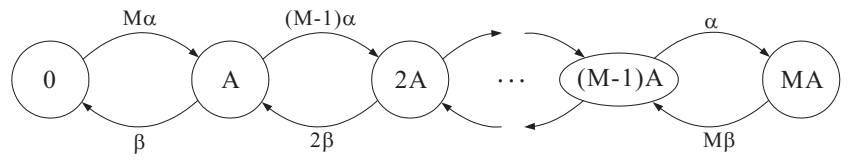

(a)

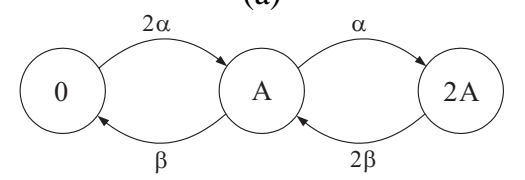

(b)

Fig. 1. Video source model (a) General video source model (b) Minisource model $(M=2)$

time traffic and nonreal-time (best-effort) traffic, respectively. The performance evaluation of uplink channel is considered. All VDTs and DTs are linked to the same base station (BS). The BS coordinates the access of each terminal for the shared channels according to a suitable channel assignment procedure. We assume that $N_{c}$ users whose data rate is $R$ kbps are supported in the system.

\section{A. Video Terminal}

Video traffic source has been modeled as a discrete-state continuous time Markov process as shown in Fig. 1(a) [9], [7]. In [9], the bit rate of video traffic is quantized into finite discrete levels $(0, A, \cdots, M A)$ and transitions between levels occur with exponential transition rates that may depend on the current level. The state transition rates of the model, $\alpha$ and $\beta$ are obtained by

$$
\begin{aligned}
& \beta=3.9 /\left(1+\frac{5.04458 N}{M}\right) \\
& \alpha=3.9-\beta
\end{aligned}
$$

where $N$ is the number of aggregated video sources and $M$ is the number of quantization levels. For simplicity of analysis, we assume the minisource model with $M=2$ and $N=1$ as shown in Fig. 1(b). VDTs are assumed to transmit packets with a rate $R$ kbps when it is in state $A$, and with a rate $2 R \mathrm{kbps}$ when it is in state $2 A$. In state 0 (null state), no packets are transmitted.

\section{B. Data Terminal}

A DT sends to the BS an access request for arrived message. The BS assigns dynamically a channel to the DT according to the chanel status. The generation of data traffic at each DT is assumed to be an independent Poisson process with a message arrival rate of $\left(\lambda_{d} / N_{d}\right) \mathrm{msg} / \mathrm{s}$, where $\lambda_{d}$ is a composite (i.e., new + retransmission) message arrival rate and $N_{d}<N_{c}$ is assumed. The data messages have an exponentially distributed length with mean value $\bar{m}$. Moreover, we assume that both the DT's access request and the BS' reply are transmitted without contention and the propagation delay is negligible. Therefore, the DT receives a channel information for a message transmission or NAK just after sending an access request. All common packet channels are assumed to have the same transmission rate $R$ kbps.

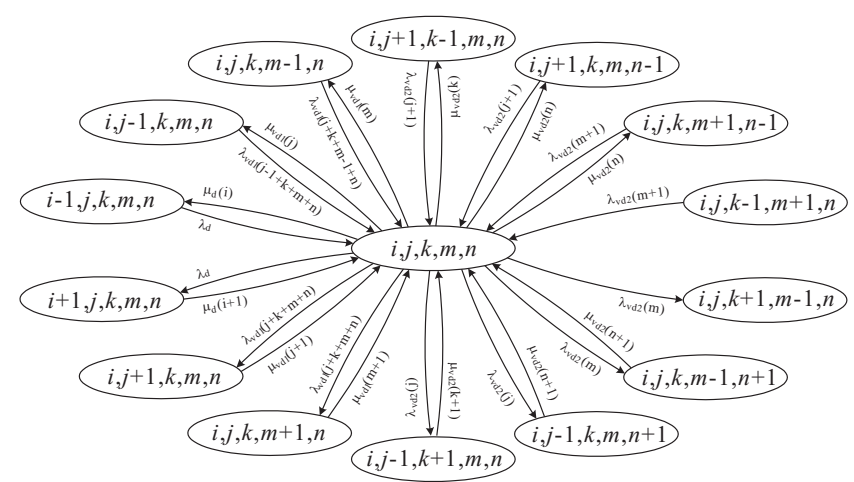

Fig. 2. State transition diagram of SCA scheme

\section{Simple Channel Assignment Scheme}

\section{A. Channel Access Control}

The BS coordinates the access of each terminal for the shared channel. It doesn't consider user terminal's traffic types. The BS keeps a channel status table to store the current status of each channel (i.e., idle or allocated). A VDT sends an access request to the $\mathrm{BS}$ as soon as the first packet is generated. Here, a state transition from null state to state $A$ occurs. And then it waits for reply of the BS. The BS tries to assign it an idle channel in the channel status table. If there is at least one idle channel, VDT's packet is transmitted. Otherwise, the packet is dropped. When the VDT transits from state $A$ to state $2 A$, it sends access request to the $\mathrm{BS}$ in order to acquire another channel. Two channels are demanded if the VDT has not an assigned channel in state $A$. That is, the VDT whose packet is dropped in state $A$ requests two channels to the BS in state $2 A$. The same channel assignment scheme is adopted to DTs. If all the channels are not in use, DT's access request is successful and a channel from the BS is assigned. If DT's request fails, the DT retries after random back off time.

\section{B. Performance Analysis}

From the system description, we model the system with SCA scheme as the Markov process. The state of the entire system, consisting of $N_{v d}$ VDTs and $N_{d}$ DTs, can be characterized by a combination of the number of user terminals and the traffic type. Therefore, the system state is denoted by

$$
s=(i, j, k, m, n), \quad(i, j, k, m, n) \in \mathcal{K}
$$

where $\mathcal{K}=\left\{(i, j, k, m, n) \mid i, j, k, m \geq 0, i+j+2 k \leq N_{c}\right\}$. The values $(i, j, k, m, n)$ is the number of user terminals that are in the data transmission mode, video packet transmission mode in state $A$, video packet transmission mode in state $2 A$, video packet dropping mode in state $A$, and video packet dropping mode in state $2 A$, respectively.

Now, let us consider the transition of the system state in equilibrium. The transition from a system state $s=(i, j, k, m, n)$ to $s^{\prime}=(u, v, w, x, y)$ occurs when the system state $s^{\prime}$ is feasible. All possible system state transition from $s$ to $s^{\prime}$ is shown in Fig. 2. From Fig. 2, state transition rates are obtained by

$$
\mu_{d}(z)=z / \bar{m}
$$




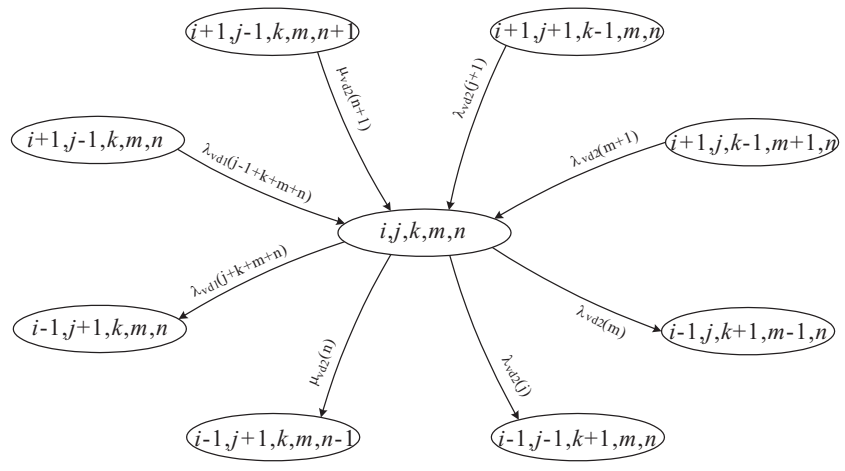

Fig. 3. State transition diagram due to preemption policy in CAP scheme

$$
\begin{aligned}
& \lambda_{v d 1}(z)=\left(N_{c}-z\right) \cdot 2 \alpha \\
& \lambda_{v d 2}(z)=z \cdot \alpha \\
& \mu_{v d 1}(z)=z \cdot \beta \\
& \mu_{v d 2}(z)=z \cdot 2 \beta
\end{aligned}
$$

Let $\pi(i, j, k, m, n)$ denote the system stationary probability of a system state $(i, j, k, m, n)$. Then we can derive $\pi(i, j, k, m, n)$ by using two following conditions for feasible $(i, j, k, m, n): 1)$ The total incoming rate of a state is equal to the total outgoing rate of the state (balance equation). 2) The sum of the steady state probabilities is 1 .

A failure to assign channels to DTs happens when all the channels are in use. Therefore, code allocation failure probability of a DT in a SCA scheme is given by

$$
P_{f a i l, d}=\left.\sum_{\forall(i, j, k, m, n) \in \mathcal{K}} \pi(i, j, k, m, n)\right|_{i+j+2 k=N_{c}} .
$$

A VDT may drop a packet when a VDT enters into state $A$ or state $2 A$. Dropping probability in state $A, P_{d r o p, v d 1}$, and that in state $2 A, P_{d r o p, v d 2}$, are calculated by

$$
\begin{aligned}
P_{d r o p, v d 1} & =\left.\sum_{\forall(i, j, k, m, n) \in \mathcal{K}} \pi(i, j, k, m, n)\right|_{m>0} \\
P_{d r o p, v d 2} & =\left.\sum_{\forall(i, j, k, m, n) \in \mathcal{K}} \pi(i, j, k, m, n)\right|_{n>0} .
\end{aligned}
$$

\section{Channel Assignment Scheme with Preemption}

\section{A. Channel Access Control}

We propose a channel assignment scheme with preemption policy for data transmission to reduce the failure rate in channel assignment to a VDT. When a VDT in null state enters into state $A$, it achieves a channel from the BS if there is at least one idle channel or even if all the channels are in use, at least one of them has been devoted to a data message transmission. In the latter case, the BS preempts DT's message transmission and assigns the channel of the preempted DT to the VDT. We assume that this preemption procedure occurs only one at a time. Therefore, the preemption procedure occurs in the following cases.

(i) If all the channels are in use, a DT is preempted in the following cases; 1) when a VDT in null state generates a packet, 2) when, in state $2 A$, a VDT in the dropping mode enters into state $A, 3$ ) when, in state $A$, a VDT in the transmission mode transits to state $2 A$.

(ii) If only one channel is idle, in state $A$, a VDT in the dropping mode enters into state $2 A$.

\section{B. Performance Analysis}

We model the system with preemption policy for data transmissions by using the same Markov process as that of the SCA scheme. The system state is denoted by (3). However, four preemption cases as shown in Fig. 3 should be considered. Let $P\left(s^{\prime} \mid s\right)$ denote the state transition rate $\operatorname{Pr}\{s=$ $\left.(i, j, k, m, n) \rightarrow s^{\prime}=(u, v, w, x, y)\right\}$. Then, the general transition rates are the same as that of the SCA scheme and the transition rates of four preemption cases are given by (12).

Let $\pi(i, j, k, m, n)$ denote the system stationary probability of a system state $(i, j, k, m, n)$. Then we can derive $\pi(i, j, k, m, n)$ for feasible $(i, j, k, m, n)$ by using the same method as that of the SCA scheme.

The dropping probabilities of video packets in state $A$ and $2 A$ are the same as the equations (10) and (11), respectively. However, a failure to assign channels to DTs happens when the BS preempts the data transmission to assign its code to other VDT as well as when all the codes are in use. In Fig. 3, the preemption probability $P_{p r e, d}$ that the BS preempts data transmissions is obtained by (13).

$$
\begin{aligned}
& P\left(s^{\prime} \mid s=(i, j, k, m, n)\right) \\
& \quad= \begin{cases}\lambda_{v d 1}(j+k+m+n) & , \text { if } s^{\prime}=(i-1, j+1, k, m, n), i>0, i+j+2 k=N_{c} \\
\mu_{v d 2}(n) & \text { if } s^{\prime}=(i-1, j+1, k, m, n-1), i>0, i+j+2 k=N_{c} \\
\lambda_{v d 2}(j) & \text { if } s^{\prime}=(i-1, j-1, k+1, m, n), i>0, i+j+2 k=N_{c} \\
\lambda_{v d 2}(m) & \text { if } s^{\prime}=(i-1, j, k+1, m-1, n), i>0, i+j+2 k=N_{c}-1 .\end{cases}
\end{aligned}
$$

$$
\begin{aligned}
P_{p r e, d}= & \sum_{\forall(i, j, k, m, n) \in \mathcal{K}}\left[\left\{\frac{\lambda_{v d 1}(i+k+m+n)}{\lambda_{d}}+\frac{\mu_{v d 2}(n)}{\lambda_{d}}+\frac{\lambda_{v d 2}(j)}{\lambda_{d}}\right\}\right. \\
& \left.\left.\cdot \pi(i, j, k, m, n)\right|_{i+j+2 k=N c, i>0}+\left.\frac{\lambda_{v d 2}(m)}{\lambda_{d}} \cdot \pi(i, j, k, m, n)\right|_{i+j+2 k=N c-1, i>0}\right] .
\end{aligned}
$$




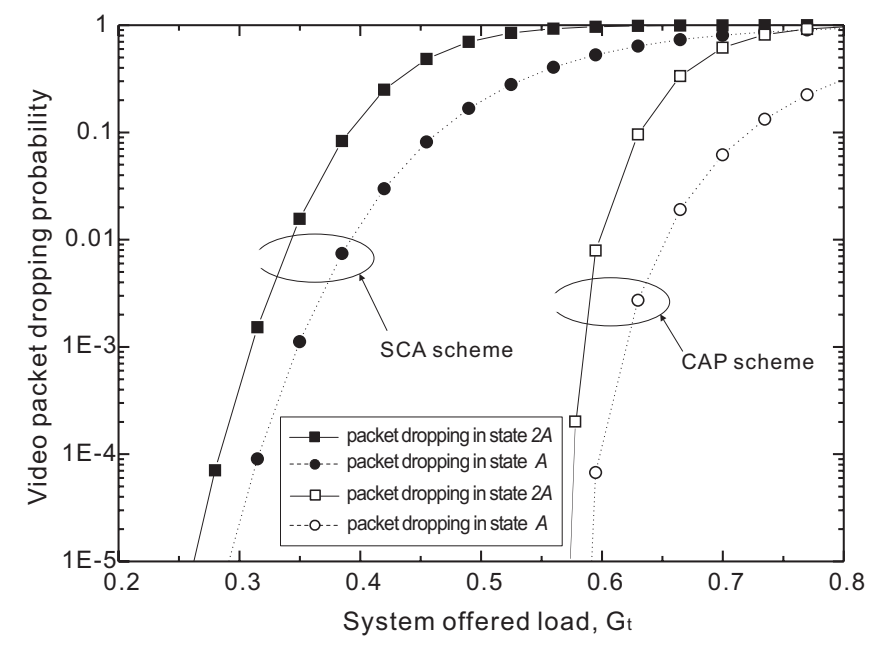

Fig. 4. Video packet dropping probability vs. system offered load when $\gamma_{v d}=$ 0.3

The probability that a DT fails in a message transmission is the sum of the probability that all the codes are in use and the probability that the data transmission is preempted. The failure probability is given by

$$
\begin{gathered}
P_{\text {fail }, d}=\left.\sum_{\forall(i, j, k, m, n) \in \mathcal{K}} \pi(i, j, k, m, n)\right|_{i+j+2 k=N_{c}} \\
+P_{p r e, d}
\end{gathered}
$$

where $P_{p r e, d}$ is given by (13).

\section{NumERICAL RESUlTS}

In the video traffic mode as shown in Fig. 1(b), the probability that a VDT is in state $A$ or $2 A$ is as follows:

$$
\begin{aligned}
P_{A} & =2 \alpha \beta /(\alpha+\beta)^{2} \\
P_{2 A} & =\alpha^{2} /(\alpha+\beta)^{2} .
\end{aligned}
$$

Therefore, when the number of active VDTs is $N_{v d}$, total offered load $G_{v d}$ of video packets is given by

$$
\begin{aligned}
G_{v d} & =\left[\frac{1}{\alpha+\beta} \cdot P_{A}+\frac{1}{2 \beta} \cdot P_{2 A}\right] \cdot N_{v d} \\
& =\frac{4 \alpha \beta^{2}+\alpha^{2}(\alpha+\beta)}{2 \beta(\alpha+\beta)^{3}} \cdot N_{v d} .
\end{aligned}
$$

Then, we define a system offered load $G_{t}$ as follows:

$$
G_{t}=\left(G_{v d}+G_{d}\right) / N_{c}
$$

where $G_{d}$ is the total data offered load and is calculated as $G_{d}=$ $\lambda \cdot \bar{m}$. The mean data message delay $D$, defined as the number of access tries needed to transmit a data message successfully, is given by

$$
D=\sum_{x=1}^{\infty} x \cdot\left(P_{f a i l, d}\right)^{x-1}\left(1-P_{f a i l, d}\right)=\frac{1}{1-P_{f a i l, d}}
$$

We assume that $N_{c}=32$ users whose data rate is $R=60$ kbps are supported in the system. The mean data message length

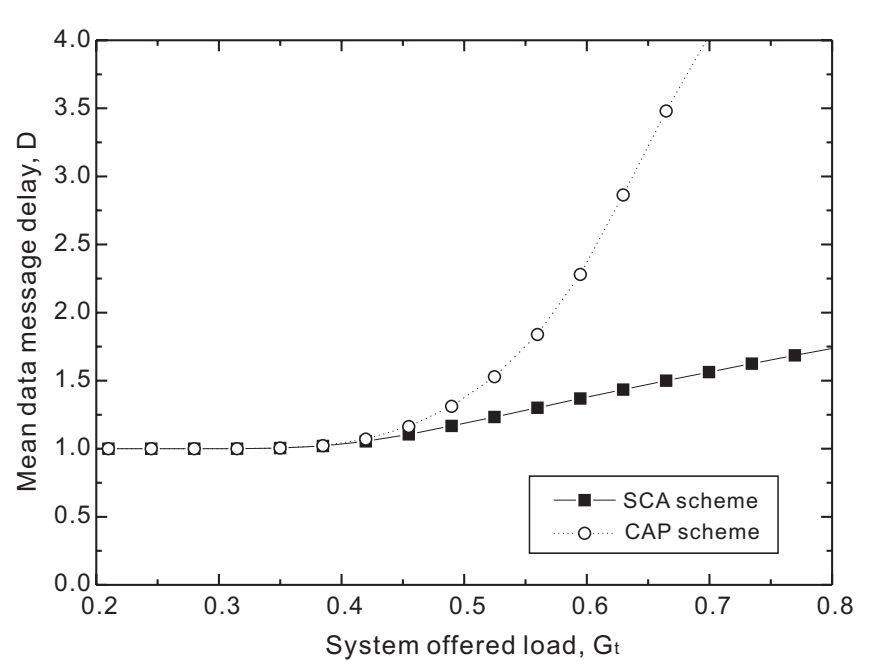

Fig. 5. Mean data message delay vs. system offered load when $\gamma_{v d}=0.3$

is assumed to be 0.064 second under $60 \mathrm{kbps}$ channel considering that the length of a request packet in WWW service is about 480 bytes [10].

First, when the ratio $\gamma_{v d}$ of the video traffic to the total traffic is 0.3 (i.e., $\left.\gamma_{v d}=G_{v d} /\left(G_{v d}+G_{d}\right)\right)$, the system performances between SCA and CAP scheme are compared in Fig. 4 and Fig. 5 in view of video packet dropping probability and mean data message delay. Fig. 4 shows the video packet dropping probability and Fig. 5 shows the mean data message delay as a function of the system offered load, respectively. As shown in Fig. 4, the video packet dropping probability exponentially increases as the system offered load increases. VDTs in state $2 A$ drop more packets than VDTs in state $A$ since a VDT in state $2 A$ needs two channels to transmit a message. In the SCA scheme, the BS doesn't consider user's traffic type and assigns the channels to users according to the channel status. In the CAP scheme, however, a failure in channel assignment to a VDT occurs only in case that all channels are used by VDTs. That is, the video packet dropping probability is not related with the data offered load but related with only the video packet offered load. Therefore, video packets are less frequently dropped and the data message delay is longer in the CAP scheme than in the SCA scheme. When the QoS requirement of VDTs is that the packet dropping probability is lower than 0.01 (i.e., $P_{d r o p}<0.01$ ), the system offered load that can be handled with satisfying the QoS requirement of a video service is about 0.6 in the CAP scheme and about 0.34 in the SCA scheme. That is, the system capacity of the proposed CAP scheme increases by 1.7 times compared with the SCA scheme in view of the video communication when $\gamma_{v d}=0.3$. However, as shown in Fig. 4, the data message delay of the CAP scheme is longer than that of the SCA scheme. This is a disadvantage of the CAP scheme. However, considering that the data traffic is delay tolerant and the system is operated under $G_{t}=0.6$ due to the QoS requirement of video communication, the negative factor is reduced.

When the number of active VDTs is 15 and 17 , the system performance is evaluated in Fig. 6 and Fig. 7. Fig. 6 shows the video packet dropping probability and Fig. 7 shows the mean data message delay as a function of the data offered load, respec- 


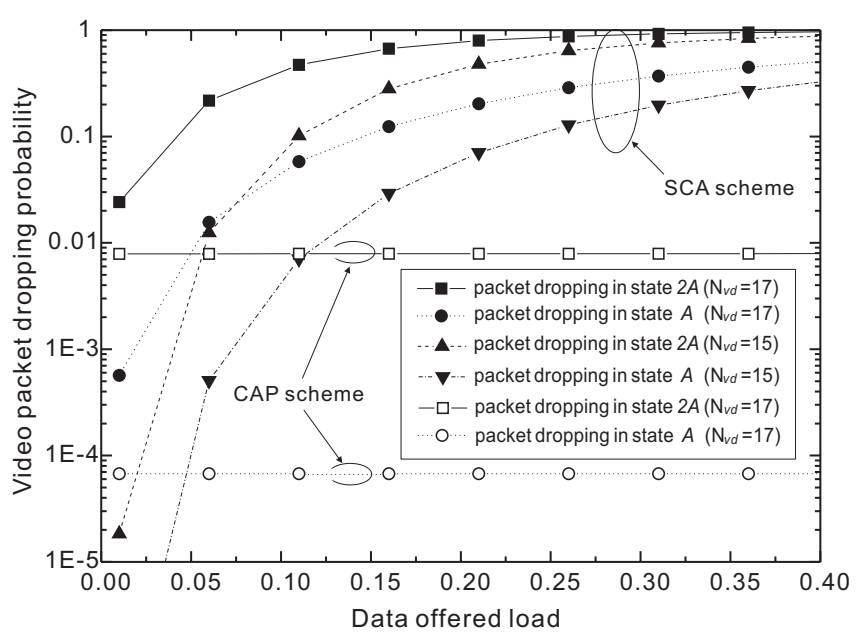

Fig. 6. Video packet dropping probability vs. data offered load

tively. If the number of active VDTs is less than or equal to $N_{c}$, the dropping of video packets doesn't occur in the CAP scheme since the BS uses the preemption policy for data transmissions. Therefore, when $N_{v d}=15$, the video packet dropping probability is zero in the CAP scheme. Moreover, the video packet dropping probability in the CAP scheme is constant value since the dropping probability is not related with the data offered load. However, in the SCA scheme, the video packet dropping probability increases as the data offered load increases since the BS doesn't consider user's traffic type. As shown in Fig. 7, the data message delay of the CAP scheme is longer than that of SCA scheme as the data offered load increases. However, considering that the system with CAP scheme satisfies the QoS requirement of VDTs and can support the data service, we can say that the proposed CAP scheme is suitable as an access control scheme for integration of video/data transmissions over the shared channel.

\section{CONCLUSIONS}

In this paper, an access control scheme based on preemption has been proposed for CDMA systems carrying the real-time video traffic and nonreal-time data traffic. A 5-state Markov process was used to model the system operation. The video packet dropping probability and mean data message delay were derived to evaluate the performances of the proposed scheme and simple channel assignment scheme without preemption policy, respectively. The results show that in the simple channel assignment scheme, the video packet dropping probability is dependent on the data offered load as well as the number of VDTs. However, in the proposed scheme, the video packet dropping probability is independent on the data offered load and is only dependent on the number of VDTs. Due to preemption policy for data transmissions, the mean data message delay increases more faster in the proposed scheme than in the simple channel assignment scheme. This increment of the data message delay, however, may be tolerable considering that the data traffic is delay tolerant.

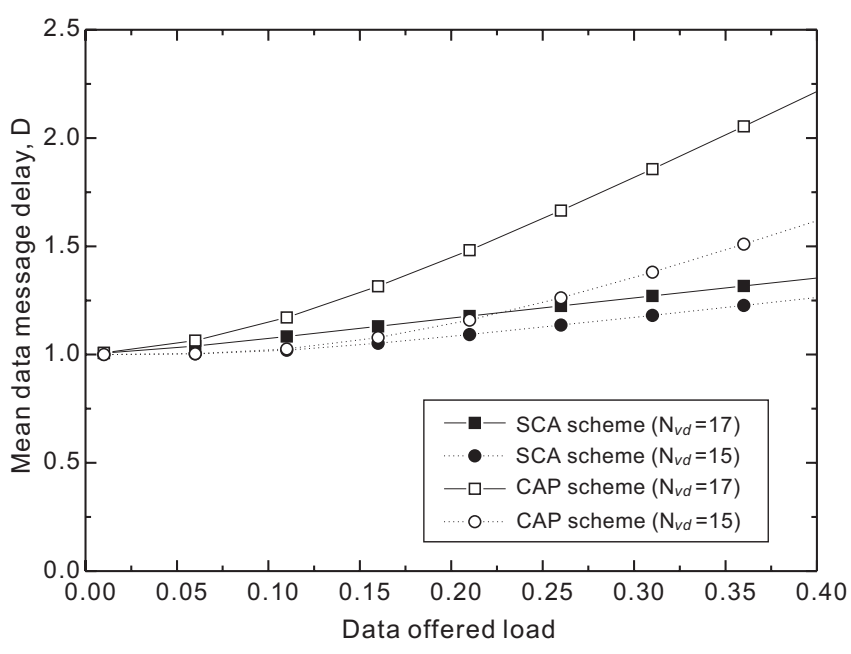

Fig. 7. Mean data message delay vs. data offered load

\section{REFERENCES}

[1] R. Fantacci and L. Zoppi, "A combined reservation random access polling protocol for voice-data transmissions in a wireless packet network," IEEE Trans. Veh. Technol., vol. 48, no. 2, pp. 652-662, Mar. 1999.

[2] J. W. So and D. H. Cho, "Access scheme for integrated voice/data transmissions over common packet channel in 3GPP," IEEE Commun. Letter, vol. 5, no. 2, pp. 46-48, Feb. 2001

[3] W. Yue and Y. Matsumoto, "Output and delay process analysis for slotted CDMA wireless communication networks with integrated voice/data transmission," IEEE J. Select. Areas Commun., vol. 18, no. 7, pp. 12451253, Jul. 2000.

[4] A. Sampath and J. M. Holtzman, "Access control of data in integrated voice/data CDMA systems: benefits and tradeoffs," IEEE J. Select. Areas Commun., vol. 15, no. 8, pp. 1511-1526, Oct. 1997.

[5] R. Pichna and Q. Wang, "A medium-access control protocol for a cellular packet CDMA carrying multirate traffic," IEEE J. Select. Areas Commun., vol. 14 , no. 9, pp. 1728-1736, 1996.

[6] N. Dimitriou and R. Tafazolli, "Quality of services for multimedia CDMA,” IEEE Commun. Magazine, vol. 38, no. 7, pp. 88-94, Jul. 2000.

[7] R. Fantacci and S. Nannicini, "Multiple access protocol for integrated of variable bit rate multimedia traffic in UMTS/IMT-2000 based on widebad CDMA,” IEEE J. Select. Areas Commun., vol. 18, no. 8, pp. 1441-1454, Aug. 2000.

[8] L. F. Akyildiz and et al., "Medium access control protocols for multimedia traffic in wireless networks," IEEE Nework, vol. 13, no. 4, pp. 39-47, July/August 1999.

[9] B. Maglaris, D. Anastassiou, P. Sen, G. Karlson, and J. D. Robbins, "Performance models of statistical multiplexing in packet video communications," IEEE Trans. Commun., vol. 36, no. 7, pp. 834-844, Jul. 1988.

[10] N. Dimitriou and R. Tafazolli, "Resource management issues for UMTS," IEE 3G Mobile Communication Technologies, pp. 401-405, 2000. 